September 4, 2007

\title{
HOMEOMORPHIC BERNOULLI TRIAL MEASURES AND ERGODIC THEORY
}

\author{
R. DANIEL MAULDIN AND ANDREW YINGST
}

\begin{abstract}
We survey the some of the main results, ideas and conjectures concerning two problems and their connections. The first problem concerns determining when two Bernoulli trial measures are homeomorphic to each other, i.e. when one is the image measure of the other via a homeomorphism of the Cantor space. The second problem concerns the following. Given a positive integer $k$ characterize those Bernoulli trial measures $m$ for which there is a homeomorphism preserving $m$ and which has exactly $k$ ergodic measures with $m$ being one of them. We will also discuss some of the history leading to these problems.
\end{abstract}

A measure on a Cantor space is taken to mean a probability measure on the Borel subsets of a space homeomorphic to Cantor space which gives non-empty open sets positive measure and gives points measure zero. That is, all measures are assumed to be full, non-atomic probability measures. In this paper, $\mathcal{C}$ is a $\equiv$ tor space means it is a topological space homeomorphic to $\{0,1\}^{N}$ provided with the product topology where $\{0,1\}$ has the discrete topology. Some particular representations of the Cantor space will hold our attention. We are interested in two problems. One is to determine when two such measures or two measures of a given type, $\mu$ on a Cantor space $X$, and $\nu$ on a Cantor space $Y$ are "homeomorphic," i.e., when is there a homeomorphism $h$ of $X$ onto $Y$ such that $\mu=\nu \circ h^{-1}$ ? The second problem concerns the ergodic properties of such measures. When is there a homeomorphism $h$ preserving $\mu$ for which $\mu$ is the unique ergodic measure or when is $\mu$ one of exactly $k$ ergodic measures for $h$ ? Besides presenting some new results and recounting some previously obtained, we will indicate some of the origins of these problems particularly as they concern Bernoulli trial measures on $\{0,1\}^{\mathbb{N}}$ or $\{0,1\}^{\mathbb{Z}}$.

2000 Mathematics Subject Classification. Primary 37B05; Secondary 28D05, $28 \mathrm{C} 15$.

Key words and phrases. homeomorphic measures, Cantor space, good measure.

The research of the first author was partially supported by NSF grant DMS0700831 . 
One of the main sources for our problems came from the foundational 1941 work of Oxtoby and Ulam [13]. A particular case of one of their main theorems is the following:

Theorem 1. Let $n \geq 2$ and consider the space $H_{\lambda}\left([0,1]^{n}\right)$ consisting of all autohomeomorphisms of the cube which preserve $\lambda$, Lebesgue measure on $[0,1]^{n}$. As $H_{\lambda}\left([0,1]^{n}\right)$ is a dense-in-itself $G_{\delta}$ set in the space of all automorphisms of the cube, it is itself a Polish space. The set of all elements of $H_{\lambda}\left([0,1]^{n}\right)$ which are ergodic with respect to Lebesgue measure forms a co-meager set in this space.

Thus, Oxtoby and Ulam showed that among the measure preserving homeomorphisms the ergodic ones are generic. At that time essentially the only known ergodic flows were irrational rotations on tori and the geodesic flows on spaces of negative curvature. Their result has been extended to manifolds and many other spaces. There is an excellent book on the subject by Alpern and Prasad [3].

One of the main ingredients for proving Theorem 1 is their characterization of those measures on the cube which are homeomorphic to Lebesgue measure.

Theorem 2. Let $n$ be a positive integer. Let $\mu$ be a probability measure defined on the Borel subsets of $[0,1]^{n}$. Then $\mu$ is homeomorphic to $\lambda$ if and only if $\mu$ gives every nonempty open set positive measure, $\mu$ is non-atomic, and $\mu$ gives the boundary of the cube measure zero.

Thus, if we consider the equivalence relation on the space of probability measures induced by two measures being homeomorphic, their theorem characterizes the measures equivalent to Lebesgue measure. This theorem has been extended to the Hilbert cube [12], and manifolds [3], and to Lebesgue measure on the Baire space, consisting of the irrational numbers in the unit interval, [10].

On the other hand the situation for the Cantor space turns out to be quite different. The Cantor space is "rigid." At first sight this may seem surprising. For example, it is amusing to show the following fact

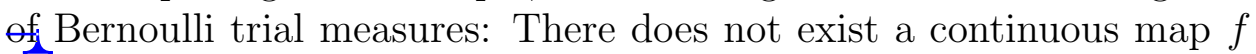
of $\{0,1,\}^{\mathbb{N}}$ to itself such that $\mu_{1 / 2}=\mu_{1 / 4} \circ f^{-1}$. Indeed, $\mu_{r}$, the measure generated by independent Bernoulli trials with probability of success $r$, is homeomorphic to $\mu_{1 / 2}$ if and only if $r=1 / 2$. (To be clear, in this paper $\mu_{r}$ denotes the Bernoulli trial measure on $\{0,1\}^{\mathbb{N}}$ of weight $r$ : the unique probability measure for which digits are independent and each has probability $r$ of being ' 1 '. This is defined for all $0 \leq r \leq 1$.) In fact, we have: 
Theorem 3. ([9],[5]) Let $r$ be rational, transcendental, or an algebraic integer of degree 2. Then $\mu_{r}$ is homeomorphic to $\mu_{s}$ if and only if $r=s$ or $r=1-s$.

In 1988, it was shown that there is a nontrivial homeomorphism class of product measures.

Theorem 4. [11]. Let $r$ be the solution of

$$
r^{3}+r^{2}-1=0
$$

lying in the open interval $(0,1)$. The Bernoulli trial measures generated by the four distinct numbers $r, r^{2}, 1-r$ and $1-r^{2}$ are all homeomorphic to each other.

Recently, Yingst completely characterized when two Bernoulli trial measures are homeomorphic, [16]. However, the following question remains unanswered.

Question 5. Is it true that if $0<r<1$, then there are only finitely many $s$ so that $\mu_{s}$ is homeomorphic to $\mu_{r}$ ?

Remark. In [16], Yingst points out that if $r$ is an algebraic integer, then the answer to question 5 is yes. However, even in this case it is still unknown how many there are. For example, it is unknown how many product measures are homeomorphic to $\mu_{r}$ when $r$ is the solution in $[0,1]$ to $r^{3}+r^{2}-1=0$.

Next, we discuss two properties a measure on the Cantor space may have which are important for both of our main problems, goodness and refinability.

Definition 6. Let $\mu$ be a measure on a Cantor space $\mathcal{C}$.

$A$ clopen set $V$ is good when for any clopen set $U$ with $\mu(U)<\mu(V)$, there is a clopen subset $U^{\prime} \subset V$ with $\mu(U)=\mu\left(U^{\prime}\right)$. The measure $\mu$ is good when every clopen set is good.

$A$ clopen set $V$ is refinable when given clopen sets $U_{1}, U_{2}, \ldots, U_{n}$ with $\sum_{j=1}^{n} \mu\left(U_{j}\right)=\mu(V)$, there is a partition $\left\{U_{1}^{\prime}, \ldots, U_{n}^{\prime}\right\}$ of $V$ into clopen sets with $\mu\left(U_{j}^{\prime}\right)=\mu\left(U_{j}\right)$ for $1 \leq j \leq n$. The measure $\mu$ is refinable if every clopen set is refinable. The measure $\mu$ is weakly refinable if the clopen set $\mathcal{C}$ is refinable, and if every clopen set can be partitioned into (finitely many) refinable clopen sets.

Note that good implies refinable, and refinable implies weakly refinable. The notions of goodness and refinability arose in very different contexts. The term "good" was coined by Ethan Akin, when examining uniquely ergodic transformations. The two directions of the following theorem were proved by Glasner and Weiss in [7] and by Akin in [1]. 
Theorem 7. Let $\mu$ be a measure on Cantor space. Then $\mu$ is good if and only if there is a uniquely ergodic, minimal homeomorphism of Cantor space for which $\mu$ is the uniquely preserved measure.

On the other hand, the term "refinable" was coined by Dougherty, Mauldin and Yingst in [6] when considering the problem of when two measures on Cantor space might be homeomorphic. There, the following theorem was shown in the refinable case, and this was generalized to the weakly refinable case in [2] by Akin, Dougherty, Mauldin and Yingst.

Theorem 8. Let $\mu$ and $\nu$ be weakly refinable measures on Cantor space. If $\mu$ and $\nu$ have the same clopen values set, then there is a homeomorphism of Cantor space $h$ so that $\mu \circ h=\nu$.

Further, if $\mu$ is refinable, and if $\left\{C_{1}, \ldots, C_{n}\right\}$ and $\left\{D_{1}, \ldots, D_{n}\right\}$ are clopen partitions of Cantor space so that $\mu\left(C_{i}\right)=\nu\left(D_{i}\right)$ for each $i$, then $h$ may be chosen so that $h\left(D_{i}\right)=C_{i}$ for each $i$.

Here, the clopen values set of a measure $\mu$ on Cantor Space is $\{\mu(E$ $E$ is clopen $\}$. The clopen values set of $\mu$ will be denoted by $S(\mu)$.

In [6], Dougherty, Mauldin and Yingst characterized goodness and refinability for Bernoulli trial measures.

Theorem 9. Let $0<r<1$. The measure $\mu_{r}$ is refinable if and only if $r$ is transcendental, or there is an integer polynomial $R$ with $R(0) \in\{-1,1\}, R(1) \in\{-1,1\}$, and $R(r)=0$. Further, $\mu_{r}$ is good if and only if $r$ is algebraic, and there is such an $R$ which has only one root in $(0,1)$.

Note that above we may assume $R$ is the irreducible polynomial polynomial of $r$, where 'irreducible' is taken over the ring $\mathbb{Z}[x]$. So for example $2 x^{2}-1$ is irreducible, but $4 x^{2}-2$ is not, since $2\left(2 x^{2}-1\right)$ is a factorization into two non-units. In this sense, the irreducible polynomial of $r$ is unique up to sign.

The question of how weak refinability relates to refinability and goodness has not been well understood. Historically, the definition of weak refinability was chosen as the minimum property for which Theorem 8 could be proved, but this has been applied only to simplify an argument that two refinable measures are homeomorphic: Given a refinable measure, $\mu$, to show that $\mu \approx \nu$ it is sufficient to show that $S(\mu)=S(\nu)$ and that $\nu$ is weakly refinable. But, since $\mu$ and $\nu$ are homeomorphic, we actually have the stronger statement that $\nu$ is refinable. this leads to the following:

Question 10. Are the refinability and weak refinability equivalent? 
We conjecture that they are not equivalent, but the following new theorem shows that they are equivalent for $\mu_{r}$.

Theorem 11. The Bernoulli trial measure $\mu_{r}$ is weakly refinable if and only if it is refinable.

Before proving this theorem, we recall some of the main tools for working with Bernoulli trial measures. Recall that if $E$ is a clopen set in $\{0,1\}^{\mathbb{N}}$, then $E$ is expressible in the form $\cup_{j=1}^{k}\left[w_{j}\right]$, where $\left[w_{j}\right]$ represents the set of all sequences in $\{0,1\}^{\mathbb{N}}$ which begin with a word $w_{j}$ over the alphabet $\{0,1\}$. Since the clopen cylinder $\left[w_{j}\right]$ can be expressed as the disjoint union $\left[w_{j} 0\right] \cup\left[w_{j} 1\right]$ we assume without loss of generality that the cylinders $\left[w_{j}\right]$ comprising $E$ are disjoint and that the words $w_{j}$ have a common length $n$. Among those cylinders of length $n$ with exactly $k 1$ 's, each has $\mu_{r}$ measure $r^{k}(1-r)^{n-k}$, and the number of them used in the expression of $E$ is an integer between 0 and $\left(\begin{array}{l}n \\ k\end{array}\right)$. This motivates the following definition:

A polynomial $p \in \mathbb{Z}[x]$ is called a partition polynomial if there is some $n \geq 0$ for which $p$ may be expressed in the form

$$
p(x)=\sum_{k=0}^{n} c_{k} x^{k}(1-x)^{n-k},
$$

where each $c_{k}$ is an integer with $0 \leq c_{k} \leq\left(\begin{array}{l}n \\ k\end{array}\right)$. With the discussion of clopen sets above, we see easily that if $E$ is a clopen set in $\{0,1\}^{\mathbb{N}}$, then there is a partition polynomial $p$ which gives the Bernoulli trial measure of $E: p(x)=\mu_{x}(E)$ for all $x \in[0,1]$. Likewise given a partition polynomial, there is such a clopen set. We refer to $p$ as the partition polynomial associated with $E$, $_{\lambda} E$ is a clopen set associated with $p$. (Note that $p$ is determined by $E$, but $E$ is not uniquely determened by $p$ unless $p=0$ or $p=1$.) We let $\mathcal{P}$ denote the set of all partition polynomials, and if $0 \leq r \leq 1$, we let $\mathcal{P}(r)$ denote $\{p(r): p \in \mathcal{P}\}$. So $\mathcal{P}(r)$ is the clopen values set of $\equiv$ measure $\mu_{r}: \mathcal{P}(r)=S\left(\mu_{r}\right)$.

The following statements of partition polynomials are proved by Dougherty, Mauldin and Yingst in [6] and by Yingst in [16]

Theorem 12. If $p$ is a polynomial with integer coefficients, then $p$ is a partition polynomial if and only if $p$ satisfies $0<p(x)<1$ for all $x \in(0,1)$, or $p=0$ or $p=1$.

If $C$ is a clopen set in $\{0,1\}^{\mathbb{N}}$ whose associated partition polynomial is $p$, and if $q$ is an integer polynomial satisfying $0<q<p$ on the interval $(0,1)$, then there is a clopen set $C^{\prime} \subset C$ whose associated partition polynomial is $q$. 
We now prove Theorem 11. This argument is a generalization of one of the directions of Theorem 9.

Proof. It's clear that weakly refinable implies refinable. Also, if $r$ is transcendental then $\mu_{r}$ is both refinable and weakly refinable. So, suppose that $r$ is an algebraic number for which $\mu_{r}$ is weakly refinable. Let $R(x)$ be the irreducible polynomial of $r$. Let $k$ be a positive integer such that $R(x)^{2}+1<\left(\frac{1}{x(1-x)}\right)^{k}$ holds on $(0,1)$. This will hold, as $\frac{1}{x(1-x)} \geq 4$ on $(0,1)$, while $R$ is continuous on $[0,1]$.

Consider the clopen set $C=\left[1^{k+1} 0^{k}\right]$. This set has $x^{k+1}(1-x)^{k}$ as its associated partition polynomial. By weak refinability, there is a refinable clopen set $D \subseteq C$. Let $f(x)$ be the partition polynomial associated with $D$. Then $0<f(x) \leq x^{k+1}(1-x)^{k}$ holds on $(0,1)$. So we have that

$$
R(x)^{2}<\frac{1}{x^{k}(1-x)^{k}}-1<\frac{1}{x^{k}(1-x)^{k}}-\frac{f(x)}{x^{k}(1-x)^{k}}
$$

holds on $(0,1)$.

Next we argue that there is some $j>k$ so that the inequality

$$
(1-x)^{j}<(1-x)^{k} R(x)^{2}+\frac{f(x)}{x^{k}}
$$

holds on $(0,1)$. Note that $R(x)$ is irreducible and isn't $x$, so we cannot have $R(0)=0$. First, for sufficiently large $j$, we will have that $(1-x)^{j}<$ $(1-x)^{k} R(x)^{2}$ holds for $x$ in $(0, \delta)$ for sufficiently small $\delta>0$. If $R(0)^{2}>1$, then this is trivial; if $R(0)^{2}=1$, then we may note that for large $j$, the derivative of $(1-x)^{j}$ at 0 will be less than that of $(1-x)^{k} R(x)^{2}$ at zero. So for some large $j$, the desired inequality holds on $(0, \delta)$, and will continue to hold on $(0, \delta)$ for larger $j$. Next we observe that the right-hand side of the above inequality is positive on $(0,1)$, so we have that the right-hand side may decrease to 0 as $x \rightarrow 1^{-}$ but can do so only at most polynomial speed. So for large $j$, we will have that $(1-x)^{j}$ decreases to zero even faster, and we will have that the desired inequality holds on $\left(\delta^{\prime}, 1\right)$ for some small $\delta^{\prime}>0$, and again for a fixed $\delta^{\prime}$ this will continue to hold for even larger $j$. Finally, the right-hand side is positive on $(0,1)$, so is greater than $\epsilon>0$ on $\left[\delta, \delta^{\prime}\right]$. For large $j$, we will have that $(1-\delta)^{j}<\epsilon$, and so we will have that the decreasing function $(1-x)^{j}$ is less than $\epsilon$ on $\left[\delta, \delta^{\prime}\right]$. So we now have for large $j$ that the desired inequality holds on $(0,1)$, and we may assume that $j>k$. 
Combining our inequalities we have

$$
(1-x)^{j-k}-\frac{f(x)}{x^{k}(1-x)^{k}}<R(x)^{2}<\frac{1}{x^{k}(1-x)^{k}}-\frac{f(x)}{x^{k}(1-x)^{k}}
$$

on $(0,1)$. Manipulating this yields

$$
0<x^{k}(1-x)^{k} R(x)^{2}+f(x)-x^{k}(1-x)^{j}<1-x^{k}(1-x)^{j}<1 .
$$

Let $g(x)$ be the second expression of the above inequality. By the first statement of Theorem 12, we have that $g(x)$ is a partition polynomial, and so $g(r)$ is a clopen value for $\mu_{r}$. Also, the clopen $\left[1^{k} 0^{j}\right]$ witnesses that $r^{k}(1-r)^{j}$ is a clopen value for $\mu_{r}$. We have the equation $g(r)+$ $r^{k}(1-r)^{j}=f(r)=\mu_{r}(D)$. Since $D$ is refinable, there is a clopen partition $\left\{C_{1}, C_{2}\right\}$ of $D$ so that $\mu_{r}\left(C_{1}\right)=g(r)$, and $\mu_{r}\left(C_{2}\right)=r^{k}(1-r)^{j}$. Let $h$ be the partition polynomial of $C_{2}$. (So $h(r)=r^{k}(1-r)^{j}$.) Since $C_{2} \subseteq D \subseteq\left[1^{k+1} 0^{k}\right]$, it follows that $0<h(x) \leq x^{k+1}(1-x)^{k}$ holds on $(0,1)$. Thus $h$ has a root at 0 of multiplicity at least $k+1$. We may let $\hat{h}(x)=\frac{h(x)}{x^{k}}$, and $\hat{h}$ is a polynomial with $\hat{h}(0)=0$. We have $\hat{h}(r)=(1-r)^{j}$. Therefore, $h(x)-(1-x)^{j}$ is an integer polynomial with a root at $r$, and by Gauss' lemma there is an integer polynomial $Q(x)$ so that $h(x)-(1-x)^{j}=Q(x) R(x)$. Evaluating this at 0 yields $0-1=Q(0) R(0)$, so we must have $R(0)= \pm 1$.

Applying the same argument to the measure $\mu_{1-r}$, which is homeomorphic to $\mu_{r}$ and is thus also weakly refinable, we see that the irredicible polynomial $\hat{R}$ of $1-r$ has $\hat{R}(0)= \pm 1$. But $\hat{R}(x)=R(1-x)$, so we have $R(1)= \pm 1$. By Theorem 9, we have that $\mu_{r}$ is refinable.

The results we have stated give rise to the following statement: Let $R(x)$ be an irreducible polynomial with $R(0)= \pm 1, R(1)= \pm 1$ so that $R$ has exactly one root $r$ in $(0,1)$. Then there is a uniquely ergodic minimal homeomorphism of $\{0,1\}^{\mathbb{N}}$ whose unique ergodic measure is $\mu_{r}$. Noticing this, Dan Mauldin asked whether 'exactly one' could be replaced by any finite number:

Question 13. Let $R(x)$ be an irreducible polynomial with $R(0)= \pm 1$, $R(1)= \pm 1$. If $R$ has exactly $k$ roots, $r_{1}, \ldots, r_{k}$ in $(0,1)$, does there exist a minimal homeomorphism of $\{0,1\}^{\mathbb{N}}$ with exactly $k$ ergodic measures, $\mu_{r_{1}}, \ldots, \mu_{r_{k}}$ ?

This question is one of the main motivations for this paper. A positive answer would give another natural example of transformations with exactly $k$ ergodic measures, see e.g., [4]. One indication that this question may have an affirmative answer is the following theorem, which states that for the group action on $\{0,1\}^{\mathbb{N}}$ of all homeomorphisms which preserve one of the $\mu_{r_{i}}$, the ergodic measures are precisely $\left\{\mu_{r_{i}}\right\}_{i=1}^{k}$. 
Theorem 14. Let $r$ be an algebraic number for which $\mu_{r}$ is refinable. Let $G$ be the group of all homeomorphisms of $\{0,1\}^{\mathbb{N}}$ for which the measure $\mu_{r}$ is invariant. (So $G=\left\{h \in \operatorname{Hom}\left(\{0,1\}^{\mathbb{N}}\right): \mu_{r} \circ h^{-1}=\mu_{r}\right\}$.) Then the ergodic measures for $G$ are the measures of the form $\mu_{s}$ where $s$ is an algebraic conjugate of $r$ in $(0,1)$.

(A measure said to be invariant under a group action $G$ of bimeasurable bijections of a space if it invariant under each element of $G$. Such a measure $\mu$ is said to be ergodic for $G$ if any measurable $E$ with $g(E)=E$ for all $g \in G$, we have $\mu(E)$ is 0 or 1.)

Proof. First note that $G$ contains the every homeomorphism of $\{0,1\}^{\mathbb{N}}$ which is a permutation of finitely many indices. Any measure which is invariant under $G$ is invariant under these maps, and is said to be exchangable. (In the more common definition, the random variables $\pi_{n}$, the $n$th projection maps are exchangable.) By de Finetti's theorem if the probability measure $\nu$ on $\{0,1\}^{\mathbb{N}}$ is exchangable, then there is a probability measure $m$ on $[0,1]$ so that

$$
\nu(E)=\int_{[0,1]} \mu_{x}(E) d m(x)
$$

for every clopen set $E$. (Thus, this holds for every Borel set as well.) Here $\mu_{x}$ is as usual the Bernoulli trial measure with weight $x$. In other words, an exchangable measure is some weighted average of the Bernoulli trial measures. Now, we show that there are particular maps in $G$ which allow us to conclude that a measure which is invariant under $G$ has such an integral representation using only those $\mu_{x}$ where $x$ is an algebraic conjugate of $r$.

Let $R(x)$ be the irreducible polynomial of $r$, and recall from Theorem 9 that the refinability of $\mu_{r}$ implies that $R(0)= \pm 1$ and $R(0)= \pm 1$. For sufficiently large $j$ we will have that

$$
[1-x(1-x)]^{j-1}<\frac{1}{R(x)^{2}}
$$

holds on $(0,1)$. Here, we have equality at 0 and 1 , but as before we may consider derivatives of each side at 0 and 1 and we will have for large $j$ that this inequality holds on $(0, \delta] \cup[1-\delta, 1)$. For some $\delta>0$. The right-hand side is positive on $[\delta, 1-\delta]$ and so is greater than some $\epsilon>0$ while the left-hand side decreases to zero pointwise and hence uniformly, so the inequality does hold for all large $j$. Fix some such $j>1$ and we now have

$$
R(x)^{2}[1-x(1-x)]^{j}<1-x(1-x) .
$$


Manipulating this yields

$$
0<x(1-x)+R(x)^{2}[1-x(1-x)]^{j}<1 .
$$

Let $g(x)$ be the middle expression of the above inequality and let $f(x)=$ $x(1-x)$. By the first statement of Theorem 12 we have that both $f$ and $g$ are partition polynomials, and we also have that $f(x) \leq g(x)$ holds on $[0,1]$, with equality only at roots of $r$. Let $C$ and $D$ be clopen sets associated with $f$ and $g$ respectively. We then have that $\mu_{x}(C) \leq \mu_{x}(D)$ for all $x \in[0,1]$, with equality only when $x$ is an algebraic conjugate of $r$. Since $C$ and $D$ have the same $\mu_{r}$ measure, we may apply the strong statement of Theorem 8 (using $\mu=\nu=\mu_{r}$ ) to obtain a homeomorphism $h$ on $\{0,1\}^{\mathbb{N}}$ which sends $C$ to $D$ and which preserves $\mu_{r}$.

Suppose $\nu$ is a probability measure which is invariant under $G$. We have shown that $\nu$ is expressible as integral of Bernoulli trial measures with respect to some measure $m$. Combining this with the sets $C$ and $D$, and the homeomorphism $h$ above and the fact that $\nu$ is invariant under $h$, we have

$$
\int_{[0,1]} \mu_{x}(D) d m(x)=\nu(D)=\nu\left(h^{-1}(D)\right)=\nu(C)=\int_{[0,1]} \mu_{x}(C) d m(x) .
$$

But the functions $\mu_{x}(C)$ and $\mu_{x}(D)$ satisfy $\mu_{x}(C) \leq \mu_{x}(D)$ on $[0,1]$, so the measure $m$ must be supported on those $x$ 's for which equality holds. Thus $m$ is supported on the finite set of conjugates of $r$ in $[0,1]$, and any probability measure which is invariant under $G$ is a convex combination of those $\mu_{s}$ where $s$ is an algebraic conjugate of $r$ in $(0,1)$.

We now show that each such $\mu_{s}$ is ergodic for $G$. Note that it is sufficient to show that each $\mu_{s}$ is invariant under $G$, as $G$ contains the two-sided shift, $\sigma$, and each Bernoulli trial measure is ergodic for this map. (This is a slight abuse, as $\sigma$ is a map on $\{0,1\}^{\mathbb{Z}}$, but we may use a bijection between $\mathbb{N}$ and $\mathbb{Z}$ to view $\{0,1\}^{\mathbb{N}}$ and $\{0,1\}^{\mathbb{Z}}$ as equivalent.) Let $g$ be in $G$. Then for any clopen set $E$, we may consider $p_{1}$ and $p_{2}$, the partition polynomials of $E$ and $g^{-1}(E)$, respectively. We have that $G$ preserves $\mu_{r}$, so that $\mu_{r}(E)=\mu_{r}\left(g^{-1}(E)\right)$, so that $p_{1}(r)=p_{2}(r)$. This is an integer polynomial equation satisfied by $r$, and so is satisfied by any algebraic conjugate $s$ of $r$. So we have $p_{1}(s)=p_{2}(s)$ and hence $\mu_{s}(E)=\mu_{s}\left(g^{-1}(E)\right)$. This holds for each clopen set $E$, and so holds for every Borel set, and we have that $\mu_{s}$ preserves $g$ for each $g \in G$ and each algebraic conjugate $s$ of $r$ in $(0,1)$.

It is worth noting that when showing this theorem, the only measures in $G$ required were the finite permutations of indices, and one additional measure. If we again view $\{0,1\}^{\mathbb{N}}$ and $\{0,1\}^{\mathbb{Z}}$ as equivalent, 
we may note that any finite permutation of indices is expressible as a composition of $\sigma, \sigma^{-1}$, and $\tau$ where $\sigma$ is the two-sided shift, and $\tau$ is a transposition of two consecutive symbols. Thus, there is a set of only three homeomorphisms $\{\sigma, \tau, h\}$ so that the only measures which preserve all three of these are the convex combinations of those Bernoulli trial measures associate with the algebraic conugates of $r$. This will be useful in a strategy toward answering Question 13.

In trying to answer this question, it is useful to recall the construction in the known case when $r$ has no other algebraic conjugates in $(0,1)$, or equivalently, when $\mu_{r}$ is good:

Theorem 15. (Akin) Let $\mu$ be a good measure on Cantor space. Then there is a uniquely ergodic minimal homeomorphism of Cantor space for which $\mu$ is the unique ergodic measure.

Proof. First, notice that goodness shows that if $a, b$ are in the clopen values set $S(\mu)$ with $a<b$, then $b-a \in S(\mu)$. From this, it follows that $S(\mu)$ is a countable subgroup of $[0,1]$ with addition mod 1 . Since $\mu$ is non-atomic and full, we know that this subroup is dense. Consider adjusting the topology of $[0,1]$ in the following way: replace each $x \in S(\mu) \backslash\{0,1\}$ with two values $x^{-}<x^{+}$, otherwise leaving the usual order of $[0,1]$ unchanged. Endowing this set with the order topology yields a space, $F$, which is homeomorphic to Cantor space, the clopen sets of which are finite disjoint unions of intervals of the form $\left[x^{+}, y^{-}\right],\left[0, y^{-}\right],\left[x^{+}, 1\right]$, or $[0,1]$. We allow $F$ to inherit Lebesgue measure $\lambda$ from $[0,1]$. (There are only countably many points of discrepancy, so this is well defined.) Then the clopen values set of $\lambda$ is the set of all finite sums of differences of elements of $S(\mu)$. But $S(\mu)$ is a group, so we find that $S(\mu)=S(\lambda)$. That $S(\mu)$ is a group also makes it easy to see that $\lambda$ is a good measure: Given $U, V$ clopen in $F$ with $\lambda(U)<\lambda(V)$, there is an $x \in[0,1]$ so that in the real sense we have $\lambda(V \cap[0, x])=\lambda(U)$. By the group properties we can show $x \in S(\mu)$, so $U^{\prime}=V \cap\left[0, x^{-}\right]$is clopen as desired.

So $\mu$ and $\lambda$ are good (and hence refinable) measures which have the same clopen values sets. So $\mu$ and $\lambda$ are homeomorphic.

If $S(\mu)$ contains an irrational value $\alpha$, we have that adding $\alpha \bmod$ 1 is a homeomorphism of $F$. (We have $x \in S(\mu)$ iff $x+\alpha \bmod 1$ $\in S(\mu)$, so we naturally interpret $\left(x^{+}+\alpha\right) \bmod 1$ as $(x+\alpha \bmod 1)^{+}$, and similar for $x^{-}$. We regard $0=0^{+}$and $1=0^{-}$.) By using the wellknown properties of an irrational rotation, we easily find that this map is a uniquely ergodic minimal homeomorphism, whose unique ergodic measure is $\lambda$. Such a map for $\lambda$ implies the existence of such a map for the equivalent measure $\mu$. 
In the event that $S(\mu) \subset \mathbb{Q}$, we can show that there is an odometer system with the same clopen values set as $\mu$. The most elegant construction of such a system is via an inverse limit of groups, but we present a more concrete representation for readers unfamiliar with the techniques.

Using the fact that $S(\mu)$ is a group, it can easily be shown that $S(\mu)=\left\{\frac{n}{d}: d \in D, 0 \leq n \leq D\right\}$ for some set $D$ of positive integers. We may assume that $D$ is closed under divisors, meaning that if $d_{1} \in D$ and $d_{2} \mid d_{1}$ then $d_{2} \in D$. Since $S(\mu)$ is dense in $[0,1]$, we have that $D$ is infinite. Let $\left(m_{1}, m_{2}, \ldots\right)$ be a sequence of elements of $D$ with the property that every product $m_{1} \cdot \ldots \cdot m_{n}$ is in $D$, and that every element of $D$ divides such a product. Then we let $X=\prod_{n=1}^{\infty}\left\{0,1, \ldots, m_{n}\right\}$ with the product topology, so that $X$ is a Cantor space, and we define a group structure on $X$ by "addition with carries."

The formal definition of this group is inductive: Let $x, y \in X$, and let $c_{0}=0$. (Here $\left(c_{n}\right)$ will denote the sequence of 'carries'.) For $n \geq 1$, let $(x+y)_{n}=x_{n}+y_{n}+c_{n} \bmod m_{n}$, and let $c_{n}=1$ if $x_{n}+y_{n}+c_{n} \geq m_{n}$, with $c_{n}=0$ otherwise.

The odometer map here is $\operatorname{Od}(x)=x+1=x+(1,0,0, \ldots)$. This well chosen name indicates how such a map is obtained by visualizing a car odometer which goes forever to the left. To apply $O d$ to the sequence, one would drive one mile. This example corresponds with the choice of $m_{n}=10$ for each $n$.

It is known that this system is uniquely ergodic, and its unique ergodic measure is the product of uniform measures on $\left\{0, \ldots, m_{n}-1\right\}$. It is easy to verify in this case that the clopen values set will be the set of rationals with denominators in $D$, that this measure is good, and hence that this unique ergodic measure is homeomorphic to $\mu$.

This argument began by examining the group $S(\mu)$ and constructing a good measure in a known space with the same clopen values set. The only requirements of this group were that it be countable and dense, so we have the following corollary.

Corollary 16. If $S \subseteq[0,1]$ is a countable dense subgroup of $[0,1]$ with addition modulo 1 , then there is a good measure $\mu$ on a Cantor space so that $S=S(\mu)$.

An interesting question arises from this: By Theorem 8, we know that a weakly refinable measure is determined (up to homeomorphism) by its clopen values set. By the observation above that if $\mu$ is good, then $S(\mu)$ is a countable dense subgroup of $[0,1]$, and by Theorem 16 , 
we see that a weakly refinable measure is good if and only if $S(\mu)$ is a subgroup of $[0,1]$.

Question 17. Is there a similar characterization of those $S(\mu)$ for which $\mu$ is refinable?

We have seen that a good measure can be viewed as living naturally on an odometer, or as living on the irrational rotation with some 'cuts' inserted to adjust the topology. We'd like a similar understanding for the refinable but not good Bernoulli trial measures. It is possible to show the following theorem which gives a somewhat similar, though not as clean, alternative way of viewing certain of the refinable Bernoulli measures. The proof is quite technical, and we omit it from the current paper.

Theorem 18. Suppose that $R(x)$ is an irreducible integer polynomial with $R(0)= \pm 1, R(1)= \pm 1$, and the roots of $R$ in $(0,1)$ are $r_{1}, \ldots, r_{k}$. Suppose further that there exists an integer polynomial $q$ so that $q\left(r_{1}\right)=$ $q\left(r_{2}\right)=\ldots=q\left(r_{k}\right) \notin \mathbb{Z}$. Then there is a Cantor space $\mathcal{C}$ and a good measure $m$ on $\mathcal{C}$ so that the measures $m \times \mu_{r_{j}}$ are simultaneously homeomorphic to $\mu_{r_{j}}$. That is, there is a single homeomorphism $h$ : $\mathcal{C} \times\{0,1\}^{\mathbb{N}} \rightarrow\{0,1\}^{\mathbb{N}}$ so that $\mu_{r_{j}} \circ h=m \times \mu_{r_{j}}$ for $j=1 \ldots k$.

In this theorem, $m$ is a good measure whose clopen values set consists of numbers of the form $q\left(r_{1}\right)$ where $q$ is a polynomial with $q\left(r_{1}\right)=$ $\ldots=q\left(r_{k}\right)$ and with $0<q\left(r_{1}\right)<1$. The additional hypothesis of this theorem which wasn't present in Theorem 14 is necessary to insure that such values exist.

This theorem shows a way of viewing a refinable but not good product measure on $\{0,1\}^{\mathbb{N}}$ as living on the product of $\{0,1\}^{\mathbb{N}}$ with either an odometer or what is essentially an irrational rotation. This result may also provide a foothold for constructing an almost uniquely ergodic measure, namely as a skew product which takes advantage of the well known uniquely ergodic transformation.

As an example of the hypotheses of the theorem, we can use $R(x)=$ $17 x^{2}(1-x)^{2}-1$. Then $R$ has two roots in $(0,1)$, namely

$$
r_{1}, r_{2}=\frac{1}{2} \pm \sqrt{\frac{1}{4}-\sqrt{\frac{1}{17}}}
$$

We may take $q(x)=x^{2}(1-x)^{2}$, and we'll have $q\left(r_{1}\right)=q\left(r_{2}\right)=\frac{1}{17}$. We could also use $q(x)=x(1-x)$, and have $q\left(r_{1}\right)=q\left(r_{2}\right)=\sqrt{1 / 17}$. This will be significant later, as we'll see we have some options determined by whether $q\left(r_{j}\right)$ can be chosen to be rational or irrational. 
Before continuing, we note that Theorem 18 begs an interesting question. In this theorem, we have a case of the product of a good and a refinable measure being refinable (because the product $m \times \mu_{r_{j}}$ is homeomorphic to the refinable measure $\mu_{r_{j}}$.) Is this always the case? Akin et al. show that a product of two good measures is good in [2], while the example of the above paragraph shows that Theorem 18 applies in cases when $\mu_{r_{j}}$ is refinable but not good, and so a product of a refinable measure and a good measure may not be good.

Question 19. Is a product of a refinable measure with a good measure refinable?

Is a product of two refinable measures refinable?

We now examine a construction which can be made with Theorem 18. Recall that we are interested in the possibility that there is a minimal homeomorphism $h$ of $\{0,1\}^{\mathbb{N}}$ so that the ergodic measures for $h$ are $\mu_{r_{1}}, \ldots, \mu_{r_{k}}$. Under the hypotheses of the theorem, this question is equivalent to the question of whether there is a minimal homeomorphism $h$ of $\mathcal{C} \times\{0,1\}^{\mathbb{N}}$ whose ergodic measures are $m \times \mu_{r_{1}}, \ldots, m \times \mu_{r_{k}}$. One attempt at constructing such an $h$ is the following skew product:

As before, let $\sigma, \tau$ and $h$ be three homeomorphisms of $\{0,1\}^{\mathbb{N}}$ so that the only measures which are invariant under all three of these are the convex combinations of $\mu_{r_{1}}, \ldots, \mu_{r_{k}}$. Let $\rho$ be a uniquely ergodic transformation of $\mathcal{C}$ which preserves $m$. (So $\rho$ may be either an odometer or essentially an irrational rotation.)

Now let $C_{1}, C_{2}, C_{3}$ be any clopen partition of $\mathcal{C}$, and we define our skew product on $\mathcal{C} \times\{0,1\}^{\mathbb{N}}$ as follows: $u(x, y)=(\rho(x), \sigma(y))$ for $x \in$ $C_{1}, u(x, y)=(\rho(x), \tau(y))$ for $x \in C_{2}$, and $u(x, y)=(\rho(x), h(y))$ for $x \in C_{3}$. This homeomorphism has the property that among products of measures on $\mathcal{C}$ and $\{0,1\}^{\mathbb{N}}$, the only preserved measures are the convex combinations of $m \times \mu_{r_{1}}, \ldots, m \times \mu_{r_{k}}$. To see this, first observe that $\rho$ is uniquely ergodic so any preserved product measure must be of the form $m \times \nu$ for some measure $\nu$ on $\{0,1\}^{\mathbb{N}}$. Further, $\nu$ must preserve the three maps $\sigma, \tau$, and $h$, and so $\nu$ must be a convex combination of $\mu_{r_{1}}, \ldots, \mu_{r_{k}}$.

Of course, we have no motivation for assuming that an ergodic measure of $h$ must be a product measure, but the freedom allowed in this construction seems to give many places to finish an argument. For example, in dividing $\mathcal{C}$ into three pieces, no assumptions were made, and more pieces would have been fine. In particular, we used no knowledge of the behavior of $\rho$ on $\mathcal{C}$ which may lead to a very natural such division. Another useful tool is the following theorem which will ensure that an 
invariant measure for $u$ can be disintegrated along $\mathcal{C}$. This theorem was independently shown by Maharam in [8] and Rokhlin [15].

Theorem 20. Let $X$ and $Y$ be Polish spaces, and let $\nu$ be a probability measure on $X \times Y$. Then there exist probability measures $m$ on $X$ and $\nu_{x}$ on $Y$ for each $x \in X$ so that $\mu$ can be expressed as $\mu(E)=$ $\int_{X} \nu_{x}\left(E_{x}\right) d m(x)$ for each Borel set $E \subseteq X \times Y$ if and only if the measure $\nu$ is absolutely continuous with respect to $m \circ \pi_{1}^{-1}$.

Here $\pi_{1}$ represents projection to the first coordinate, and $E_{x}$ denotes $\{y \in Y:(x, y) \in E\}$, the fiber of $E$ at $x$.

In the case of $\mu$ a measure which preserves the map $u$ above, we have that

$$
\mu \circ \pi^{-1}(E)=\mu\left(\left(h^{-1} \pi^{-1}(E)\right)\right)=\mu \circ \pi^{-1}\left(\rho^{-1}(E)\right),
$$

so $\mu \circ \pi^{-1}$ is a measure on $\mathcal{C}$ which preserves $\rho$. Since $\rho$ is uniquely ergodic, we have that $\mu \circ \pi^{-1}$ is absolutely continuous with respect to $m$ because it equals $m$.

We therefore have that a measure $\mu$ which preserves $u$ is expressible as

$$
\mu(E)=\int_{\mathcal{C}} \nu_{x}\left(E_{x}\right) d m(x) .
$$

We would like an argument that if $u$ is ergodic, then $\mu$ is a product measure, or in this context, that there is a measure $\nu$ so that $\nu_{x}=\nu$ for almost all $x \in \mathcal{C}$. We cannot argue this, but a nice beginning is as follows:

Suppose $E$ be a Borel subset of $C_{1}$, and let $D$ be a Borel subset of $\{0,1\}^{\mathbb{N}}$. We then have that

$$
\mu(D \times E)=\mu(u(D \times E))=\mu(\rho(D) \times \sigma(E)) .
$$

Using our disintegration of $\mu$, we use this to write

$$
\begin{aligned}
\int_{D} \nu_{x}(E) d m(x) & =\int_{\rho(D)} \nu_{x} \sigma(E) d m(x) \\
& =\int_{D} \nu_{\rho^{-1}(x)} \sigma(E) d m \rho^{-1}(x)=\int_{D} \nu_{\rho^{-1}(x)} \sigma(E) d m(x) .
\end{aligned}
$$

This gives that two functions $\nu_{x}(E)$ and $\nu_{\rho^{-1}(x)} \circ \sigma(E)$ have the same integral for every Borel subset of $C_{1}$, and hence are equal almost everywhere in $C_{1}$. The same arguments apply on $C_{2}$ and $C_{3}$, so we almost surely have that $\nu_{x}=\nu_{\rho^{-1}(x)} \circ \sigma$ if $x \in C_{1}$, we have $\nu_{x}=\nu_{\rho^{-1}(x)} \circ \tau$ if $x \in C_{2}$, and $\nu_{x}=\nu_{\rho^{-1}(x)} \circ h$ if $x \in C_{3}$. This gives a concrete place to begin trying to understand the invariant measures for $u$.

Finally, we observe that Theorem 18 does not apply to all nontrivially refinable measures $\mu_{r}$. Namely, the $q$ required in the theorem 
does not exist in all cases. For example, consider $R(x)=x^{3}+3 x^{2}-$ $4 x+1$. Then $R$ is irreducible as any factorization would yield a rational root, but the rational root test gives that the only possible rational roots are \pm 1 , which are not roots. We have that $R(1)=1, R(0)=1$, and $R\left(\frac{1}{2}\right)=-\frac{1}{8}$, so that $R$ has at least two roots in $(0,1)$, and since $R$ is cubic, $R$ must have exactly two roots in $(0,1)$. Let $r_{1}, r_{2}$ be the roots of $R$ in $(0,1)$, and let $r_{3}$ be the third root of $R$. Suppose $q$ is an integer polynomial with $q\left(r_{1}\right)=q\left(r_{2}\right)$. Since $r_{1}$ is an algebraic integer, we have that $q\left(r_{1}\right)$ must be an algebraic integer. Also, the polynomial $h(x)=\left(x-q\left(r_{1}\right)\right)\left(x-q\left(r_{2}\right)\right)\left(x-q\left(r_{3}\right)\right)$ has coefficients which are symmetric polynomials in $r_{1}, r_{2}, r_{3}$ and so $h$ has rational coefficients. Since $h$ has a repeated root, the factorization of $h$ into irreducible factors must split $\left(x-q\left(r_{1}\right)\right)$ and $\left(x-q\left(r_{2}\right)\right)$ which implies that $h$ has three linear factors, and $q\left(r_{1}\right)=q\left(r_{2}\right)$ must be rational. So $q\left(r_{1}\right)=q\left(r_{2}\right)$ is a rational integer, and the hypothesis of Theorem 18 is not satisfied. Even so, it is not clear in cases such as this whether the conclusion of the theorem will hold.

\section{REFERENCES}

[1] E. Akin, Good measures on Cantor space, Trans. Amer. Math. Soc. 357 (2005), 2681-2722.

[2] E. Akin, R. Dougherty, R. D. Mauldin, and A. Yingst, Which Bernoulli measures are good measures?, to appear in Colloq. Math., 49 pages.

[3] S. Alpern And V. S. Prasad, Typical dynamics of volume preserving homeomorphisms, Cambridge Tracts in Mathematics, 139, Cambridge University Press, Cambridge, 2000.

[4] I.P. Cornfeld, S.V. Fomin and Ya. G. Sinai, Ergodic Theory, SpringerVerlag, Berlin, 1982.

[5] K.J. HuAnG, Algebraic numbers and topologically equivalent measures in the Cantor set, Proc. Amer. Math. Soc. 96 (1986), 560-562.

[6] R. Dougherty, R. D. Mauldin, and A. Yingst, to appear in Trans. Amer. Math. Soc., 16 pages.

[7] E. Glasner and B. Weiss, Weak orbit equivalence of minimal Cantor systems, Internat. J. Math. 6 (1995), 559-579.

[8] D. Maharam, Decompositions of measures algebras and spaces, Trans. Amer. Math. Soc. 69 (1950), 142-160.

[9] F. J. Navarro-Bermudez, Topologically equivalent measures in the Cantor space, Proc. Amer. Math. Soc. 77 (1979), 229-236.

[10] J. C. Охтову, Homeomorphic measures in metric spaces, Proc. Amer. Math. Soc. 24 (1970), 419-423.

[11] F. J. NAvarro-Bermudez And J. C. Oxtoby, Four topologically equivalent measures in the Cantor space, Proc. Amer. Math. Soc. 104 (1988), 859-860.

[12] J. C. Oxtoby and V. S. Prasad, Homeomorphic measures in the Hilbert cube, Pac. J. Math. 77 (1978), 483-497. 
[13] J. C. Oxтовy and S. M. Ulam, Measure preserving homeomorphisms and metrical transitivity, Ann. Math. 42 (1941), 874-920.

[14] R. G. E. PINCH, Binomial equivalence of algebraic numbers, J. Indian Math. Soc. (N.S.) 58 (1992), 33-37.

[15] V. A. Rokhlin, On the fundamental ideas in measure theory, Mat. Sbornik 25 (1949), 107-150, Amer. Math. Soc. Translations 71, (1952).

[16] A. Yingst, A characterization of homeomorphic Bernoulli trial measures, to appear in Trans. Amer. Math. Soc., 29 pages.

Department of Mathematics, PO Box 311430, University of North Texas, Denton, TX 76203

E-mail address: mauldin@unt.edu

Department of Mathematics, LeConte College, 1523 Greene Street, University of South Carolina, Columbia, SC 29208

E-mail address: yingst@math.sc.edu 